\title{
Prenatal diagnosis of pulmonary artery sling associated with tracheal agenesis: a case report
}

\author{
Yanhua $\mathrm{Qi}^{1}$, huanhuan huo ${ }^{1}$, miaoyan $\mathrm{ma}^{1}$, jinfang wu ${ }^{1}$, and Baomin $\mathrm{Liu}^{2}$ \\ ${ }^{1}$ Affiliation not available \\ ${ }^{2}$ Xi'an Jiaotong University Second Affiliated Hospital
}

July 13, 2020

\begin{abstract}
Pulmonary artery sling (PAS) and tracheal agenesis (TA) are both very rare diseases. Most of PAS are associated with tracheal bronchial malformations. However, PAS associated with TA have not yet been reported so far. Here, we report one case of PSA associated with TA diagnosed prenatally in our hospital. Due to the extremely low incidence of two diseases, physicians do not have sufficient understanding of these disease, prenatal ultrasound examination found that these kinds of diseases are very challenging and confusable. Prenatal ultrasound and MRI examination of pulmonary artery branches, trachea and esophagus will provide useful information. Improving the accuracy of prenatal fetal diagnosis is helpful for perinatal management and counseling.
\end{abstract}

\begin{abstract}
Pulmonary artery sling (PAS) and tracheal agenesis (TA) are both very rare diseases. Most of PAS are associated with tracheal bronchial malformations. However, PAS associated with TA have not yet been reported so far. Here, we report one case of PSA associated with TA diagnosed prenatally in our hospital. Due to the extremely low incidence of two diseases, physicians do not have sufficient understanding of these disease, prenatal ultrasound examination found that these kinds of diseases are very challenging and confusable. Prenatal ultrasound and MRI examination of pulmonary artery branches, trachea and esophagus will provide useful information. Improving the accuracy of prenatal fetal diagnosis is helpful for perinatal management and counseling.
\end{abstract}

KEYWORDS: fetal echocardiography, pulmonary artery sling, tracheal agenesis

\section{INTRODUCTION}

Pulmonary artery sling (PAS) is a rare congenital cardiovascular malformation. In the case of PAS, the left pulmonary artery originates from the right pulmonary artery and compresses the tracheobronchus. Most of PAS are associated with tracheal bronchial malformations. There are four typical classifications of PAS, Types $1 \mathrm{~A}$ and $1 \mathrm{~B}$ are characterized by an aberrant left pulmonary artery causing tracheobronchial compression with normal bronchial pattern, Types $2 \mathrm{~A}$ and $2 \mathrm{~B}$ are characterized by an abnormal tracheal bronchial pattern, which are associated with varying degrees of tracheal stenosis ${ }^{[1]}$. However, PAS associated with tracheal agenesis (TA) is extremely rare, with no cases published in the literature. In this study, we have described a case of PAS associated with TA. Furthermore, we explore the feasibility of prenatal diagnosis of two diseases.

\section{CASE REPORT}

A healthy 32-year-old woman, G1P0, was referred to our hospital at 39 weeks of gestation. She and her husband had no family history of malformation. Prenatal ultrasound scanning displayed single umbilical 
artery, pulmonary artery sling, tracheal compression and deformation and hydramnios in other hospital at 24 weeks of gestation. The Down's screening and the cell-free fetal DNA test showed a low risk of aneuploidy. At 27 weeks of gestation, fetal magnetic resonance examination indicated that the trachea was not visible, and both the left and right tracheal branches were delicate.

Prenatal ultrasound at 39 weeks in our hospital showed that the left pulmonary artery originated from the right pulmonary artery (Fig. 1), the trachea and bronchus were not clearly displayed, and the fetal esophagus was obviously dilated to below diaphragm (Fig. 2A,2B). Combined with MRI examination, the diagnosis finally we obtained was suspicious TA combined with tracheal esophageal fistula(TEF), and PAS(aberrant left pulmonary artery).

The pregnant woman eventually give birth naturally at 41 weeks of gestation. A female neonate was born. Immediately after delivery, there was severe respiratory distress, cyanosis and no audible cry. The Apgar scores were 1 at $1 \mathrm{~min}, 7$ at $5 \mathrm{~min}$, and 9 at $10 \mathrm{~min}$, birth weight of $3460 \mathrm{~g}$. Eendotracheal intubation was attempted difficultly, but succeeded. Then a large number of gastric contents were sucked out. The neonate breathed difficultly, then transferred to neonatal intensive care unit immediately. Based on the results of gastrointestinal barium meal examination(Fig. 3) and three-dimensional reconstruction of bronchus CT(Fig. 4), the neonate was finally diagnosed with tracheal agenesis(Floyd type III) and PAS(aberrant left pulmonary artery). After counseling, the family decided to withdraw the treatment after neonate born 15 days, and the autopsy was denied.

\section{DISCUSSION}

The incidence rate of PAS among children is approximately 59 in 1 million ${ }^{[2]}$. In a fetus with PAS, there is no normal pulmonary bifurcation at the bifurcate of trachea and pulmonary artery below the aortic arch, and only the main pulmonary artery is directly connected to the arterial canal and the right pulmonary artery. Careful ultrasound examination, by slight adjustment of the probe, at the level of the pulmonary bifurcation may lead to clear images of PAS, which reveals an abnormal LPA that originates from the posterior wall of the right pulmonary artery, traveling posterior to the trachea, and entering the left lung ${ }^{[3]}$. The difficulty in the diagnosis of this case is that the relationship between the left pulmonary artery and the trachea bronchus is not clear because the trachea is not shown.

TA is also an extremely rare kind of malformation with incidence less than 1:50000 and far poorer prognosis, which will result in the fetal death due to be unable to breathe after birth ${ }^{[4]}$. Thus far, around 150 cases have been reported in literature ${ }^{[5]}$. The trachea and esophagus are developed from the embryonic primitive foregut. On the 21-26th day, the foregut is separated by the mesoderm ridge growing towards it, in this way the ventral part forms the trachea and the dorsal part forms the esophagus. If things went wrong during the growth and fusion of mesodermal ridge, it would cause various types of tracheal atresia and tracheoesophageal fistula. TA was classified into three types by Floyd et al ${ }^{[6]}$. Type I: accounting for $10 \%$, the trachea is absent except for a short distal segment with normal carina. There is a tracheoesophageal fistula connecting the distal part of the trachea to the oesophagus. Type II: covering at most $59 \%$, the trachea is absent completely; the two main bronchi join at the carina and in almost all cases there is a carino-oesopha-geal fistula. Type III: the trachea and carina are absent and each of the main bronchi join the oesophagus from either side. This clinical case belongs to Floyd type III. It is a great challenge for prenatal ultrasound examination to detect such disease ${ }^{[7]}$. During fetal development, alveoli secretes fluid under normal conditions, which forms the liquid membrane in lungs to maintain the normal development of lungs, while the fluid can communicate with amniotic fluid through trachea-larynx-oronasal cavity and then be discharged. When TA occured together with TEF, the lung fluid can be discharged into esophagus through fistula, which cause the enlargement of the esophagus, but the lungs are normal. Therefore, it is great difficulty in definitive prenatally diagnosis of $\mathrm{TA}^{[8]}$. In this case, the enlarged esophagus was detected behind the atrium of the fetus, communicating upward with the pharynx and downward with the stomach bubble through the diaphragm. The trachea was not shown by ultrasound and MRI. All of above mentioned was help to make the diagnosis of TA with TEF in prenatal. 
The prognosis for patients with PAS is largely affected by the occurrence of respiratory complications. The vascular circle formed by PAS can partially surround and compress the trachea and esophagus. If not treated in a timely manner, the mortality rate is extremely high. However, there is no vascular ring in our case, the lack of the trachea is more troubling. Airway management in TA has proven to be an insurmountable challenge in most cases resulting in infant mortality. Therefore, the prognosis of this case was very poor and subsequent treatment had to be abandoned.

\section{Conclusion}

PAS and TA are both very rare diseases, they occur simultaneously are unprecedented. Due to the extremely low incidence of PAS and TA, physicians do not have sufficient understanding of this disease, prenatal ultrasound examination found that these kinds of diseases are very challenging and confusable. Prenatal ultrasound and MRI examination of pulmonary artery branches, trachea and esophagus will provide useful information. Improving the accuracy of prenatal fetal diagnosis is helpful for perinatal management and counseling.

Reference

[1] Theadis R, Wells1John L, Gwinn1Benjamin H, et al. Reconsideration of the anatomy of sling left pulmonary artery: The association of one form with bridging bronchus and imperforate anus, Anatomic and diagnostic aspects. Journal of Pediatric Surgery. 1988;23, 892-898.

[2] Yu J-M, Liao C-P, Ge S, et al. The prevalence and clinical impact of pulmonary artery sling on school-aged children: a large-scale screening study. Pediatr Pulmonol. 2008;43:656-661.

[3] Li T, Han J, Gao S, Hao X, He Y. Prenatal diagnosis of tetralogy of Fallot associated with pulmonary artery sling: Two case reports. Echocardiography. 2019;00:1-4.

[4] H MOHAMMED, K WEST, J BEWICK, et al. Tracheal agenesis, a frightening scenario. The Journal of Laryngology \& Otology. 2016; 130: 314-317.

[5] Park BJ, Kim MS, Yang JH, Jun TG. Tracheal agenesis reconstruction with external esophageal stenting: postoperative results and complications. Korean J Thorac Cardiovasc Surg. 2015;48:439-442.

[6] Floyd J, Campbell DC Jr, Dominy DE. Agenesis of the trachea. Am Rev Respir Dis 1962; 86:557-560.

[7] De Groot-van der Mooren, Haak MC, Lakeman P, et al. Tracheal agenesis: approach towards this severe diagnosis, Case report and review of the literature. Eur J Pediatr. 2012;171:425-31.

[8] de Groot-van der Mooren Maurike D, Haak Monique C, Lakeman Phillis, et al. Tracheal agenesis: approach towards this severe diagnosis. Case report and review of the literature.Eur. J. Pediatr. 2012; 171: 425-31. 

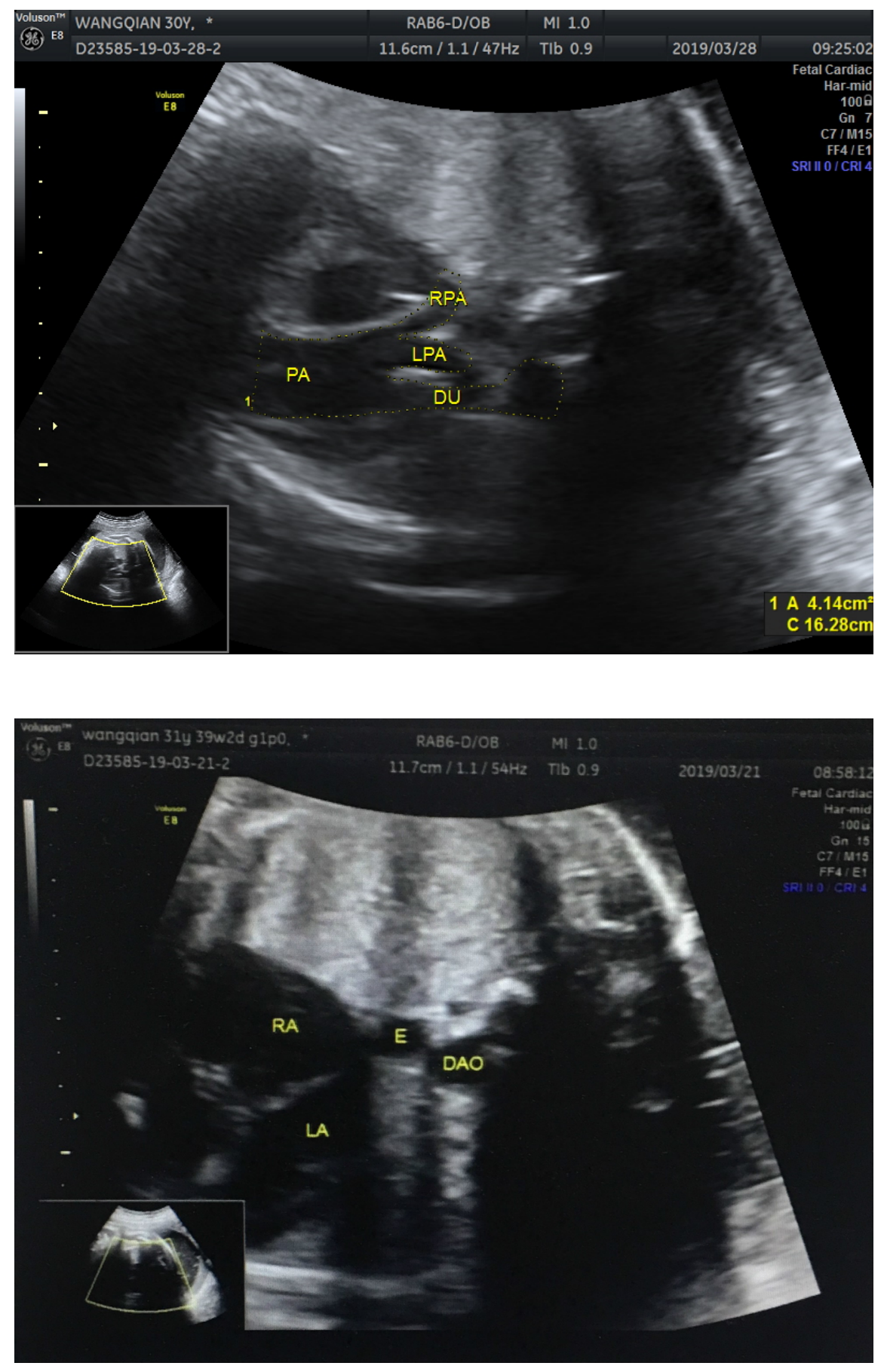

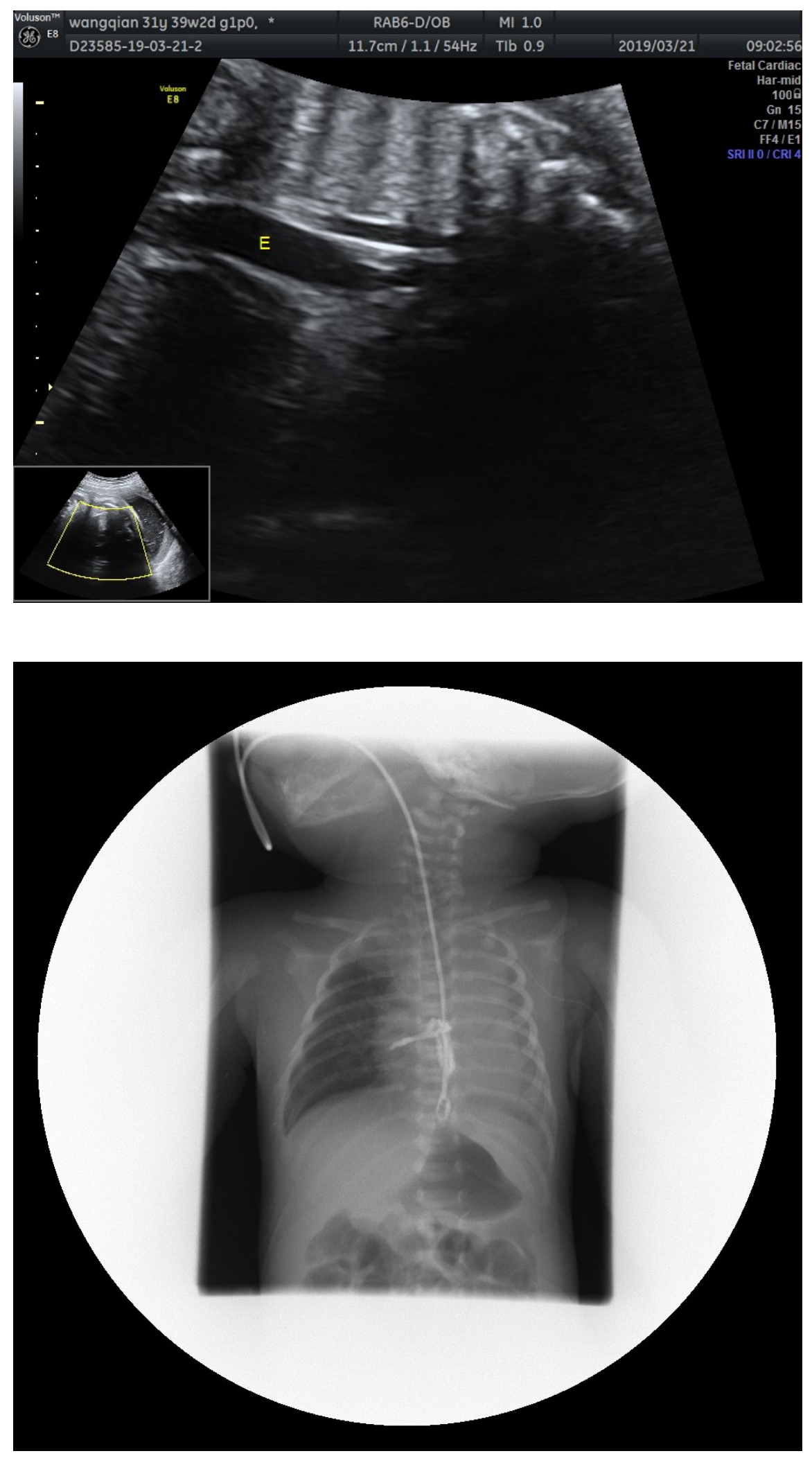


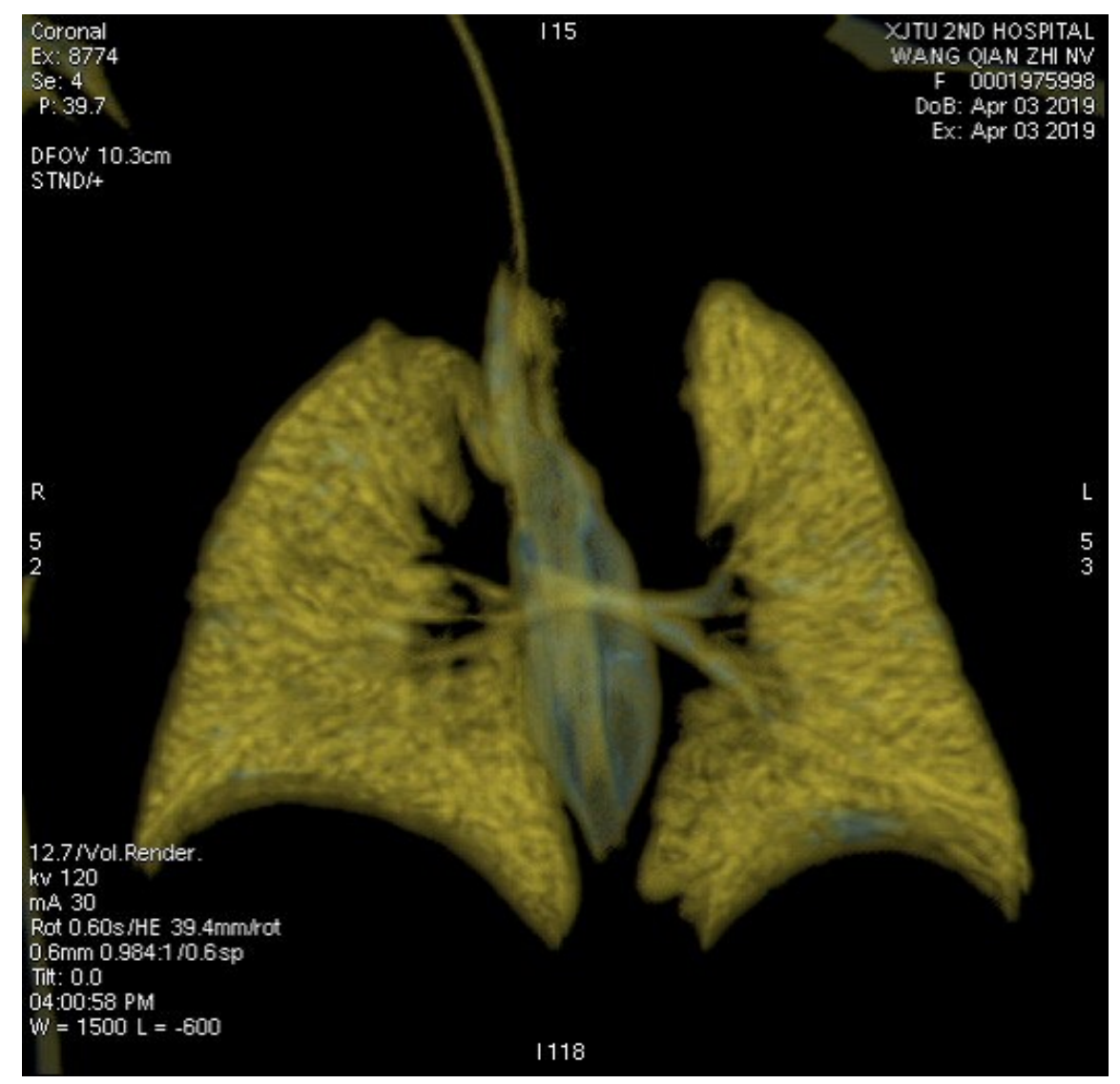

